Check for updates

Cite this: RSC Adv., 2018, 8, 29939

\title{
One-pot synthesis of graphene quantum dots and simultaneous nanostructured self-assembly via a novel microwave-assisted method: impact on triazine removal and efficiency monitoring $\dagger$
}

\author{
Beatriz Fresco-Cala, ${ }^{a}$ M. Laura Soriano, (D) *a Alice Sciortino, ${ }^{\text {bc }}$ Marco Cannas, (D) ${ }^{b}$ \\ Fabrizio Messina (D) ${ }^{\mathrm{b}}$ and Soledad Cardenas (D) *a
}

\begin{abstract}
One-step methods for fabricating green materials endowed with diverse functions is a challenge to be overcome in terms of reducing environmental risk and cost. We report a fast and easy synthesis of multifunctional materials composed of only fluorescent dots with structural flexibility and high sorption capability. The synthesis consists of a one-pot microwave-assisted reaction for the simultaneous formation of graphene quantum dots (GQDs) from organic precursors and their spontaneous self-assembly forming porous architectures. The GQD-assemblies are robust and no signs of degradation were observed with most organic solvents. The ensuing GQDs and their porous solids were fully characterized at the morphological and optical levels. Interestingly, the solid integrates both the advantages of porous materials and the nanoscale, showing a marked sorption capability towards hazardous electron-deficient triazines (112 $\mathrm{mg} \mathrm{g}^{-1}$ of sorbent). Moreover, it also exhibits optical-responsive properties based on quantum confinement when it is disassembled acting as a fluorometric sensor in alcoholic solutions. Therefore, these properties enable this novel material to became a convenient bifunctional analytical tool not only for the removal of herbicides in apolar organic solvents but also as a chemosensor to monitor their presence in polar media. This work opens very challenging possibilities of creating porous graphene-based networks for contaminant

remediation and monitoring.
\end{abstract}

Received 20th May 2018

Accepted 16th August 2018

DOI: $10.1039 / c 8 r a 04286 a$

rsc.li/rsc-advances

\section{Introduction}

Stunning progress has been recently made in developing novel carbon-based materials for a variety of different functions. Individual graphene sheets were explored for many applications although problems of random agglomeration hamper and restrict their practical use. Great attention is focused on the newly emerged family of carbon-based nanodots: they can be synthesized via a variety of straightforward oxidizing reactions

${ }^{a}$ Departamento de Química Analitica, Instituto de Química Fina y Nanoquímica, Edificio anexo Marie Curie, Campus de Rabanales, Universidad de Córdoba, E-14071 Córdoba, Spain. E-mail: scardenas@uco.es; qa2sodom@uco.es; Tel: +34 957218616

${ }^{b}$ Dipartimento di Fisica e Chimica, Università degli studi di Palermo, via Archirafi 36, 90123, Palermo, Italy

${ }^{c}$ Dipartimento di Fisica e Astronomia, Università degli studi di Catania, via Santa Sofia 64, 95123, Catania, Italy

$\uparrow$ Electronic supplementary information (ESI) available: Optical characterization of graphene quantum dot and its sorptive behaviour towards terbutryn, and tables for comparing the synthetic conditions, characteristics and roles of the GQD assembly with others already published, and of the optimized parameters of the respective decay kinetic curves and the corresponding estimated lifetimes. See DOI: 10.1039/c8ra04286a from various carbon sources, and display fascinating fluorescent features, excellent solubility and large surface area. ${ }^{1}$ Amongst them, the so-called graphene quantum dots (GQDs), ${ }^{2,3}$ which can be described as biocompatible 2-dimensional singlelayered crystalline nanostructures, are an excellent alternative for graphene in a wide variety of applications mainly in catalysis, bioimaging, drug delivery and analytical sensing. ${ }^{4-6}$ of special interest is their use as sorbent materials to minimize environmental risks related to pollutants, although their highwater solubility hampers their applicability in this research field. To avoid this shortcoming, the preparation of novel formats such as gel-like and porous materials based on carbon nanostructures has been paid great attention in the last decade.

Hence, the development of efficient synthetic procedures to prepare porous solids based exclusively on carbon nanomaterials is highly desirable. The most used approaches so far are, on the one hand, the direct growth of nanoparticles by Chemical Vapor Deposition ${ }^{7}$ which is a relatively expensive and complex technique, and in addition, synthesized solids show a significant amount of residual metal catalyst affecting to their intrinsic properties. On the other hand, porous materials can also be obtained from a nanoparticle dispersion at a critical 
concentration due to the gelation behaviour promoted by van der Waals and $\pi-\pi$ interactions between nanoparticles. ${ }^{\mathbf{8} 9}$ However, in the majority of cases the use of different additives like redox reagents or even cross-linkers are required to form the gel. ${ }^{10}$ Thus, it is still a challenge to create robust porous solids based on nanoparticles in a fast way without adding extra linkers; but indeed, it became more challenging getting in one single step both the nanoparticle synthesis and gel formation.

With the increasing use of herbicides for implementing the agricultural productivity, and their negative impact on the environment and human health, the demand of sustainable methods for their monitorization and removal has increased dramatically. ${ }^{\mathbf{1 1 - 1 3}}$ Typically, these toxic compounds are detected using very expensive instrumentation; thus, economic alternatives for its analysis are under active research. In this sense, nanotechnology has paid a crucial role since variety of nanomaterials were applied to either extract or report the presence of pesticides. ${ }^{\mathbf{1 4}}$ However, many of these nanomaterials are limited by their high aggregation and toxicity, damaging the environment. In contrast to other carbon nanomaterials, GQDs are on the rise for being considered eco-friendly and endowed with large surface area capable of interacting and quantifying pesticide; however, its use is limited by the lack of batch-tobatch reproducibility and the scarce understanding on its photoluminescence origin.

Most importantly, contaminant removal should be ideally combined with sensing capabilities. To achieve this double goal, the self-assembly of GQDs into a porous network seems to be a promising alternative since enables to incorporate the sorptive properties of such fluorescent nanodots into porous materials. While gel-like networks have been used in conjunction with presynthesized GQDs for diverse applications, ${ }^{\mathbf{1 5 - 1 8}}$ to date one-pot microwave method to achieve both the synthesis of GQDs and their simultaneous assembly getting a porous material has not been reported yet for extracting and sensing triazines.

This work reports on the formation of easy-to-fabricate porous continuous solid based on GQDs from nanoparticle precursors by a microwave-assisted organic solvent-free protocol. Thus, the synthesis of GQD containing carboxyl and amine groups at the graphitic edges is conducted by a bottomup methodology. This synthetic route represents a fast way for preparing porous materials based on GQDs. We provide evidence of the excellent batch-to-batch reproducibility of these aerogels that resulted to be very robust in apolar media. In polar solvents, these GQD-architectures are disassembled into welldispersed GQDs that displayed excellent fluorescence. After characterizing the main structural and optical properties of the porous GQD-architecture and their individual component, GQDs, their promising sorption features towards triazines as well as their fluorometric sensing behaviour were evaluated. In this respect, it is shown the double function of this novel nanostructured material for the systematic extraction and also the monitorization of the remediation efficiency towards triazines. This novel material with two roles (sorbent/sensing) is of great relevance for creating real-world methods for reduction and evaluation of contaminant attenuation rates of setting remediation targets.

\section{Experimental section}

\section{Reagents}

Phosphoric acid (85 wt\% in $\mathrm{H}_{2} \mathrm{O}$ ), urea (99\%), glucose, (99\%), sodium hydroxide, ethanol (99\%) methanol, hexane, and chloroform were purchased from PANREAC, S.A.U. (Barcelona, Spain). Ethylenediaminetetraacetic acid (99\%), calcium nitrate tetrahydrate $(\geq 99 \%)$, sodium nitrate $(\geq 99 \%)$, magnesium nitrate (98\%), cobalt(II) nitrate hexahydrate (98\%), terbutryn, prometryn and simetryn were supplied from Sigma-Aldrich (Madrid, Spain). Ultrapure water was obtained through Millipore system for high purification.

\section{Preparation and optimization of the graphene quantum dots}

To find the optimal synthetic conditions, different protocols were performed using phosphoric acid and varying the precursor's dosages, and reaction time in a microwave reactor. Briefly, urea $(8.35 \mathrm{mmol})$ and glucose $(0.02 \mathrm{mmol})$ were mixed in presence of $\mathrm{H}_{3} \mathrm{PO}_{4}(17 \mathrm{mmol})$ and $2 \mathrm{~mL}$ of double distilled water and heated under microwave radiation at different temperatures (ranging 140, 150 and $160{ }^{\circ} \mathrm{C}$ ) for 10 and $20 \mathrm{~min}$. The residue obtained was treated with double distilled water and centrifuge at $13000 \mathrm{rpm}$ for removal of any big aggregates. Yellow-brownish solutions were obtained with blue photoluminescent features. All the GQD solutions were neutralized and purified by precipitation with ethanol at low temperatures.

\section{Formation and self-assembly of GQDs into a porous material}

Nanostructured GQD-assemblies were formed in situ by upscaling the precursors needed for the preparation of GQD by microwave irradiation. Urea $(16.65 \mathrm{mmol})$ and glucose $(2.8$ mmol) were heated for $20 \mathrm{~min}$ with $\mathrm{H}_{3} \mathrm{PO}_{4}(32 \mathrm{mmol})$, as described in the GQD preparation protocol. This procedure resulted in foam-like gel formation over the course of the reaction, however gels proved relatively weak and an ageing process is required for getting a strong porous material. Solubility experiments demonstrated the resistance to apolar solvents like alkanes whilst it dissolved in a polar media. The content of amine groups was determined in triplicate using the Kaiser test kit. The isoelectric points and conductivity of the disassembled GQDs from the aerogel in water were calculated at $25{ }^{\circ} \mathrm{C}$ using a Malvern Zetasizer NanoZSP in the range of $\mathrm{pH}$ 2.18.5. The conductivity of this GQDs at $\mathrm{pH} 6$ resulted to be 10.2 $\mathrm{mS} \mathrm{cm} \mathrm{cm}^{-1}$ and the isoelectric point of 5.11 .

\section{Spectroscopic and microscopic characterization}

Fluorescence intensity and the maximum excitation and emission wavelengths of GQDs in polar solvents were evaluated. Each measurement was carried out three times, and the average maxima fluorescent bands were found at $\lambda_{\mathrm{ex}}=370$ and $\lambda_{\mathrm{em}}=$ $416 \mathrm{~nm}$, using $2 \mathrm{~nm}$ of excitation and emission slit widths. Measurements were performed with a PTI QuantaMaster ${ }^{\mathrm{TM}}$ spectrofluorometer.

The absorption measurements were carried out on aqueous solution of GQDs (at $1.32 \mathrm{~g} \mathrm{~L}^{-1}$ ) by a double spectrophotometer 
(JASCO V-560) in the $200-800 \mathrm{~nm}$ range in a $1 \mathrm{~cm}$ quartz cuvette. The photoluminescence spectra were recorded by a laser-excited photoluminescence setup. Both the steady state and the timeresolved luminescence spectra were recorded on an intensified CCD camera while exciting the sample by a tunable laser system which includes an optical parametric oscillator pumped by a Q-switched Nd:YAG laser (5 ns pulses at $10 \mathrm{~Hz}$ repetition rate). To perform time-resolved measurements, the camera records the emission spectra after variable delays respect to the laser pulse during a fixed time window of $0.5 \mathrm{~ns}$. Instead, to perform steady-state measurements, the camera integrates the spectra obtained within a temporal window extending from before the photoexcitation to some hundreds of nanoseconds after photoexcitation. The spectral resolution is $4 \mathrm{~nm}$ and the accuracy on the lifetimes is about $0.2 \mathrm{~ns}$.

Raman of the dry solid material was performed with Integrated AFM/Confocal/Raman spectrometer (alpha500; WITec $\mathrm{GmbH}$ ) with a laser of $532 \mathrm{~nm}$. IR spectra were recorded using a Bruker Tensor37 attenuated total reflection Fourier transform infrared (ATR-FT-IR). Microscopic images of GQDs were performed using a TEM microscope model JEOL JEM 2010; the samples were prepared by dropping diluted solutions of GQDs on a TEM grid. For SEM characterization, the aerogels were dried under vacuum at room temperature for 2 days, coated with $3 \mathrm{~nm}$ of chromium using a Cressington 328 ultra high resolution EM coating system, and imaged using an FEI Helios Nano Lab Dual Beam microscope in immersion mode, with typical beam settings of $1.5 \mathrm{kV}$ and $0.17 \mathrm{nA}$.

\section{Sorption capacity of porous GQDs-assemblies}

Three herbicides were selected as representative pollutants to evaluate the sorption capacity of the aerogel. Standard solutions at various concentration in the range of $1-180 \mathrm{mg} \mathrm{L}^{-1}$ were prepared. Experiments were performed by introduction of spherical blocks of the aerogel (ca. $11 \mathrm{mg}$ ) into a glass vial containing the standard solutions in hexane $(10 \mathrm{~mL})$ at $25^{\circ} \mathrm{C}$. Sorption experiments were performed by a batch equilibration method after one hour, and gas chromatography/mass spectrometry unless mentioned otherwise. The sorption capacities were calculated based on the differences of the concentrations of analyte before and after the extraction procedure. Nitrogen porosimetry was used to calculate the surface area (Quantachrome ${ }^{\circledR A S i Q w i n}{ }^{\mathrm{TM}}$-Automated Gas Sorption).

\section{Chromatographic analyses}

Gas chromatographic/mass spectrometric analyses (GC/MS) were carried out on a gas chromatograph (Varian CP-3800)mass spectrometer (Varian $1200 \mathrm{MS} / \mathrm{MS}$ ) working under single quadrupole mode and with an electron multiplier detector. The gas chromatograph was equipped with a fused silica capillary column VF-5 $\mathrm{ms}(30 \mathrm{~m} \times 0.25 \mathrm{~mm}$ inner diameter) coated with $5 \%$ phenyl-95\% dimethylpolysiloxane (film thickness $0.25 \mu \mathrm{m}$ ) (Sigma-Aldrich) to separate the six analytes. System control and data acquisition was achieved with an HP1701CA MS ChemStation software.
The GC oven was programmed as follows: the initial temperature, $40{ }^{\circ} \mathrm{C}$, was maintained for $2 \mathrm{~min}$, raised up to $170{ }^{\circ} \mathrm{C}$ at $10^{\circ} \mathrm{C} \mathrm{min}-1$ and then immediately ramped at $2{ }^{\circ} \mathrm{C} \mathrm{min}^{-1}$ up to $200^{\circ} \mathrm{C}$. The final temperature, $260^{\circ} \mathrm{C}$, was reached with a ramp of $10^{\circ} \mathrm{C} \mathrm{min}^{-1}$ and maintained for $2 \mathrm{~min}$. The injector temperature was $280^{\circ} \mathrm{C}$ and the splitless mode was selected. The injection volume, $2 \mu \mathrm{L}$ of methanol, was measured with a $5 \mu \mathrm{L}$ microsyringe (Hamilton Co., Nevada, USA). The carrier gas used was helium (6.0 grade, Air Liquide, Seville, Spain) at a flow rate of 1.0 $\mathrm{mL} \min ^{-1}$, and it was regulated by digital pressure controller. The transfer line and ionization source were maintained at $280^{\circ} \mathrm{C}$ and $250{ }^{\circ} \mathrm{C}$, respectively. The ionization mode employed in the mass spectrometer was electron impact (EI) with ionization energy of $70 \mathrm{eV}$. Mass spectra were acquired using the selected ion monitoring mode (SIM) with $\mathrm{m} / \mathrm{z}$ of 213 (simetryn), 226 (terbutryn) and 241 (prometryn) at $1 \mathrm{scan} \mathrm{s}^{-1}$. Chromatograms were acquired and processed using MS Workstation (Varian) on an AMD SemproTM Processor computer (https://www.bruker.com) which also controlled the whole system.

\section{Results and discussion}

\section{Synthesis and characterization of porous GQDs-assemblies}

We believe that the use of acids during the hydrothermal carbonization of organic systems promotes the formation of fluorescent nanodots with high quantum yield. ${ }^{19-21}$ Herein, we investigated the effect of $\mathrm{H}_{3} \mathrm{PO}_{4}$ on the formation of graphitic nanodots. Fluorescent GQDs were synthesized under hydrothermal conditions from urea and glucose in presence of phosphoric acid and adapted to a microwave reactor, instead of pyrolysis of the reagents ${ }^{22}$ for minimizing time and saving energy. The reaction conditions were extensively tested and the formation of GQDs monitored by fluorescence spectroscopy. Thus, different microwave protocols (by varying the temperature, time of reaction, ratio of precursors, up-scaling) are described hereafter. Low-scale reactions using excess of urea (69:1) versus glucose in presence of $\mathrm{H}_{3} \mathrm{PO}_{4}$ for $10 \mathrm{~min}$, at temperatures of 140,150 and $160{ }^{\circ} \mathrm{C}$ gave rise to fluorescent GQDs with emission maximum at $c a .420 \mathrm{~nm}$ by exciting at $375 \mathrm{~nm}$. However, the intensity of the fluorescence increases considerably at higher temperatures for a $10 \mathrm{~min}$ reaction. Interestingly, the emission intensity after $20 \mathrm{~min}$ of reaction at $140{ }^{\circ} \mathrm{C}$ was enough to reach the completion of the GQD formation. This approach drives to carbon nanoparticles with large quantities of oxygen functional groups located at the graphitic edges as result of the water solubility. The ensuing GQDs solubilized in polar solvents displays similar maximum emission wavelengths in water and ethanol but different fluorescence intensities (Fig. 1) possibly evidencing a donoracceptor character of GQDs. The GQD resulted to be very stable in solution at room temperature for more than four months as their fluorescence intensity remained unaltered without any signal of aggregation or sedimentation. As occurred for most of GQDs, the high GQD solubility observed following this approach evidences the presence of nitrogen and oxygencontaining ending groups at the sheet edges formed during the dehydration process under microwave radiation with 


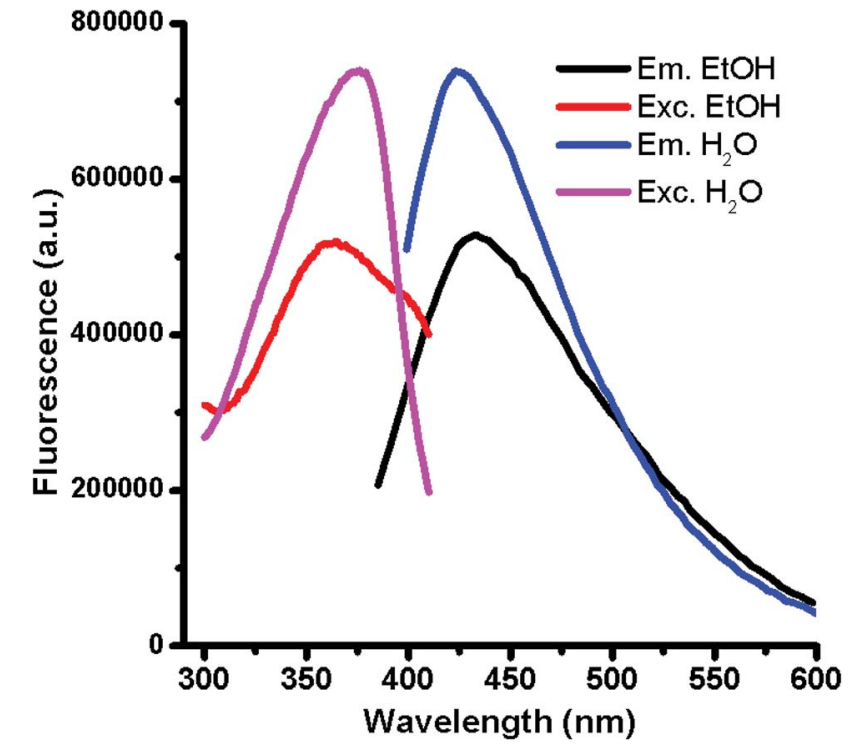

Fig. 1 Fluorescence spectra of GQDs after 20 minutes of microwave reaction. Excitation and emission curves of well-dispersed GQD in water and ethanol are shown $\left(1 \mathrm{mg} \mathrm{mL}^{-1} \cdot \lambda_{\mathrm{ex}}=375 \mathrm{~nm}\right.$. Slits at $\left.2 \mathrm{~nm}\right)$.

a reductive acid $\left(\mathrm{H}_{3} \mathrm{PO}_{4}\right)$. The formation of the graphitic structures occurs via dehydration and subsequent degradation of the precursors at $140{ }^{\circ} \mathrm{C}$ under microwave radiation.

Interestingly, it was found that besides the GQD generation an in situ gel formation occurs spontaneously when upscaling the reaction and increasing the carbon precursor proportion (ratio of $6: 1$ of urea/glucose) for a period of $20 \mathrm{~min}$ at $140{ }^{\circ} \mathrm{C}$ with a high reproducibility, as sketched in Scheme 1 . Table S1 $\dagger$ shows an overview of the synthetic protocols followed for the reaction. For characterizing the surface of the aerogels, Kaiser tests were assessed with positive results indicating that free amine groups were onto the graphitic nanostructures in a concentration of 100 $\mu \mathrm{mol} \mathrm{g}{ }^{-1}$. It was found that the assembly of aerogels with the newly formed GQDs occurs below its isoelectric point, 5.11, which would affect to possible carboxyl acids and ammonium cations attached at the GQD edges. Because the formation of a gel-like structure spontaneously occurs in the course of the reaction, it is expected that gel formation undergoes by self-assembly of ending groups holding from the graphitic nanosheets. Notably, it was observed that a low shrinkage (less than $5 \%$ ) occurred during drying. The GQD-assemblies resulted to be stable in most of organic media like apolar solvents (chloroform and hexane) and polar protic (ethanol) and aprotic (acetone, ethyl acetate) organic solvents except for dimethyl sulfoxide (Fig. S1 $\dagger$ ) due to its higher dispersing ability (larger dipole moment). However, after treatment with water and alcohols, the tridimensional structures were completely dissolved only by the simple action of ultrasound frequencies, meaning that non-covalent interactions like hydrogen bonding may be mainly responsible for the GQD self-assembly. It is noteworthy to mention that other non-covalent forces such as $\pi$ stacking interactions between nanosheets may reinforce this solid network.

The optical and structural characterization of GQDs derived from the spontaneously aerogel (sample 5 of Table S1†)
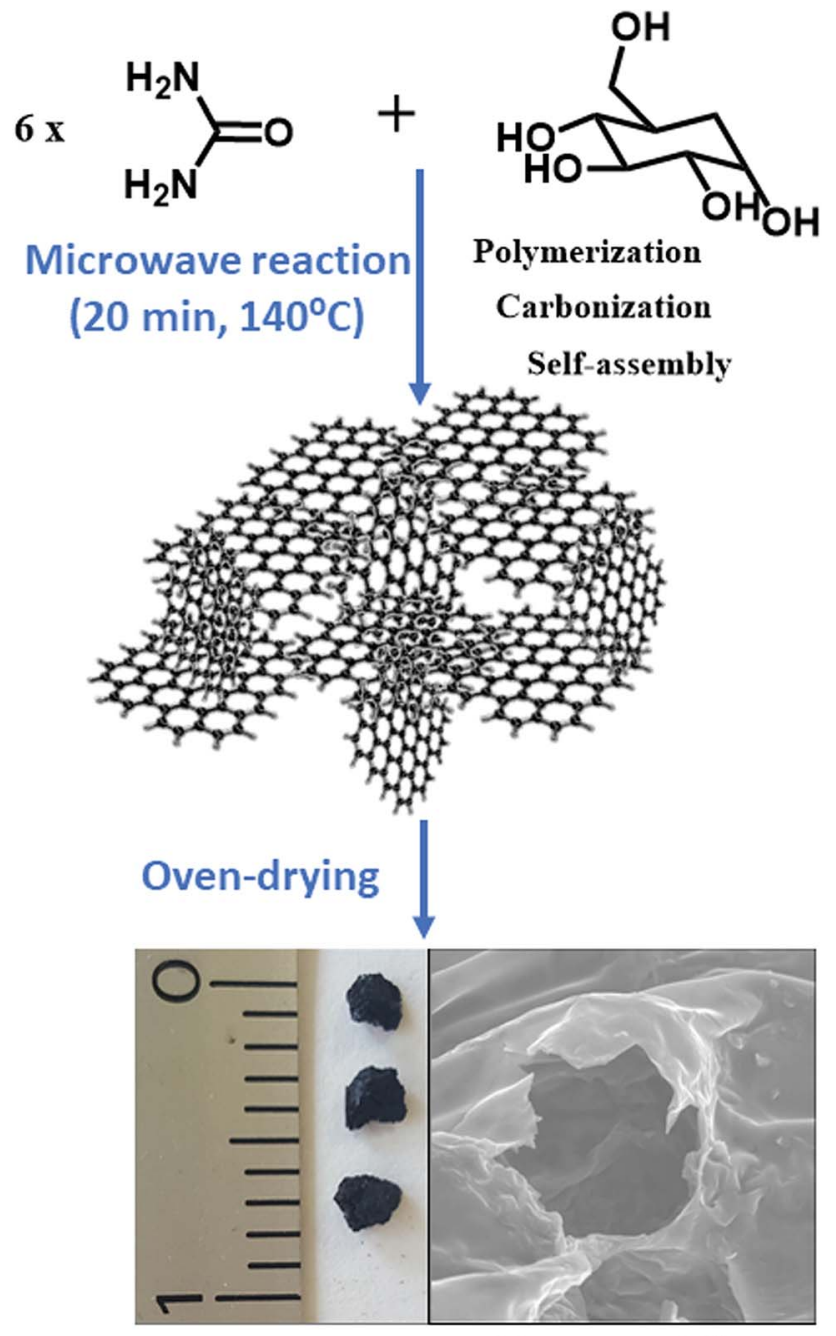

Porous GQD-self-assemblies

Scheme 1 Synthetic procedure of the GQD-assemblies via microwave-assisted one-pot reaction.

disassembled in polar solvents were investigated. The optical absorption spectrum of these GQDs dispersed in water (Fig. S2 $\dagger$ ) displays three shoulders at 257, 277 and $370 \mathrm{~nm}$ over a continuous unstructured absorption extending from the deepUV to the visible. This unstructured absorption is very common for graphitic and carbonaceous nanodots although slightly dependent on the synthetic route. ${ }^{21,23}$ The two lower-wavelength bands at 257 and $277 \mathrm{~nm}$ are mostly due to transitions of the $\mathrm{sp}^{2}$ cores, confirming the formation of GQDs. In contrast, the UV band are usually associated to surface states derived from $n-\pi^{*}$ transitions linked to the presence of double bonds of carbon and heteroatoms, like $\mathrm{C}=\mathrm{O}$, at the edges of the graphitic sheets.

Next question that comes up is to see the fluorescence behaviour of GQDs. Most importantly, the GQDs display a tunable fluorescence; Fig. 2 shows the normalized emission spectra of GQDs. In particular, exciting at any wavelength shorter than $390 \mathrm{~nm}$, their emission is non-tunable and always peaks at $416 \mathrm{~nm}$. At longer wavelengths $(400-500 \mathrm{~nm})$, the sample showed the typical visible tunability of carbon 


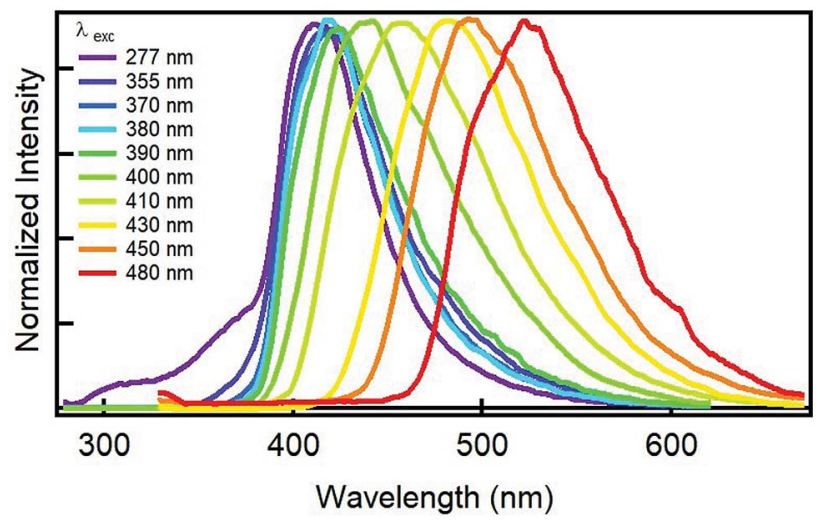

Fig. 2 Normalized emission spectra of an aqueous solution of GQDs derived from the GDQ-assembly (sample 5 of Table S1†) upon exciting at different wavelengths (indicated in the legend).

nanoparticles, ${ }^{24-26}$ whereby the emission maximum shifts from 416 to $520 \mathrm{~nm}$ while excitation wavelength changes from 390 to $480 \mathrm{~nm}$. The aforementioned fluorescence changes and the fullwidth at half maximum emission below $100 \mathrm{~nm}$ may indicate a narrow variety in the sizes and in the emissive sites coming from edges defects and functional groups of the ensuing GQDs. Another important aspect concerns the GQD's efficiency to fluoresce. The quantum yield (QY) of GQD aqueous solution were measured at two wavelengths; exciting at $370 \mathrm{~nm}$, the QY resulted to be of $32 \%$, on the contrary, the QY excitation at $410 \mathrm{~nm}$ resulted to diminish to $11 \%$. The QY at $370 \mathrm{~nm}$ is very high if compared to other carbon-based nanodots, ${ }^{1}$ especially those derived from similar precursors, ${ }^{19,21,27}$ indicating the important role played by the acids in the synthesis of bright nanodots (Table S2†).

Besides, time resolved measurements at both 370 and $410 \mathrm{~nm}$ excitations were performed to investigate the decay kinetics of the emission. The kinetic behaviour are quite complex. As shown in the left panels of Fig. S3 and S4, $\uparrow$ the decays of the emission are bi-exponential and the lifetimes (reported in Tables S3 and S4 $\dagger$ ) depend on the emission wavelength. As a consequence, the emission bands undergo a redshift upon time (right panels of Fig. S3 and S4†). The average excited-state-depopulation time $\left(\tau_{\mathrm{m}}\right)$ spans from 2 to 6 ns in accordance to the emission wavelength (see Tables S3 and $\mathrm{S} 4$ ). These lifetime values of a few nanoseconds in combination with the large QYs clearly suggest that the fluorescent transitions are strongly dipole-allowed, feature very characteristic in carbon-based nanodots. ${ }^{1}$

Transmission electron microscopy (TEM) micrographs of GQDs confirm the spherical morphology of individual nanoislands with sizes below $15 \mathrm{~nm}$ and their agglomerates after dryness (Fig. 3). To know more about possible defects in the graphitic structure and the functional groups attached on it, infrared (IR) and Raman analyses were carried out. Fig. 4A shows the IR spectrum of the GQD aerogel obtained directly by attenuated total reflection (ATR). Broad peaks from $2600-3400 \mathrm{~cm}^{-1}$ are ascribed to the $\mathrm{O}-\mathrm{H}$ and $\mathrm{N}-\mathrm{H}$ vibration modes which are involved in hydrogen bonds, indicating the main forces responsible for the retention of the porous network. In addition, a strong stretching vibration mode of $\mathrm{C}=\mathrm{O}$ centered at $1700 \mathrm{~cm}^{-1}$ suggests the presence of carboxyl acid groups possibly situated at the sheet edges and adsorbed water. Peaks from 1276 to $1425 \mathrm{~cm}^{-1}$ can be attributed to $\mathrm{C}-\mathrm{N}$ and $\mathrm{C}-\mathrm{O}$ stretching vibration modes. The characteristic peaks of $\mathrm{C}-\mathrm{H}$ vibration were also observed at 2924 and $2858 \mathrm{~cm}^{-1}$, possibly due to alkyl ending chains at the edges of the nanosheets connecting the oxygen and nitrogen-containing groups. From this data, it is clearly observed that hydrogen bonding is strongly influencing the aerogel network. Furthermore, the Raman spectrum depicted in Fig. 4B shows the two distinctive peaks of graphitic structures: the typical D-band at $1373 \mathrm{~cm}^{-1}$ due to the longitudinal vibration of disorder carbon, and G-band at around $1598 \mathrm{~cm}^{-1}$ referred to the transverse graphene vibrational mode (out-phase). The atomic ratio of $\mathrm{sp}^{2} / \mathrm{sp}^{3}$ carbons, expressed as the ratio of the intensity Raman peaks $\left(I_{\mathrm{D}} / I_{\mathrm{G}}\right)$, resulted to be 0.88 . The blue-shifts and the broadening of both $\mathrm{D}$ and $\mathrm{G}$ bands presumably corroborate the creation of defect sites on the graphitic layer possibly by the doping of heteroatoms like nitrogen..$^{28,29}$

Beside the analysis of the GQDs extracted from the aerogel (Fig. 2 to 4), further characterization was performed on the aerogel itself. The textural characterization of the aerogel was performed by nitrogen sorption isotherm using a porosimeter, and the resulting surface areas were of $110 \mathrm{~m}^{2} \mathrm{~g}^{-1}$ which is acceptable if compared to other graphitic porous structures; ${ }^{29-31}$
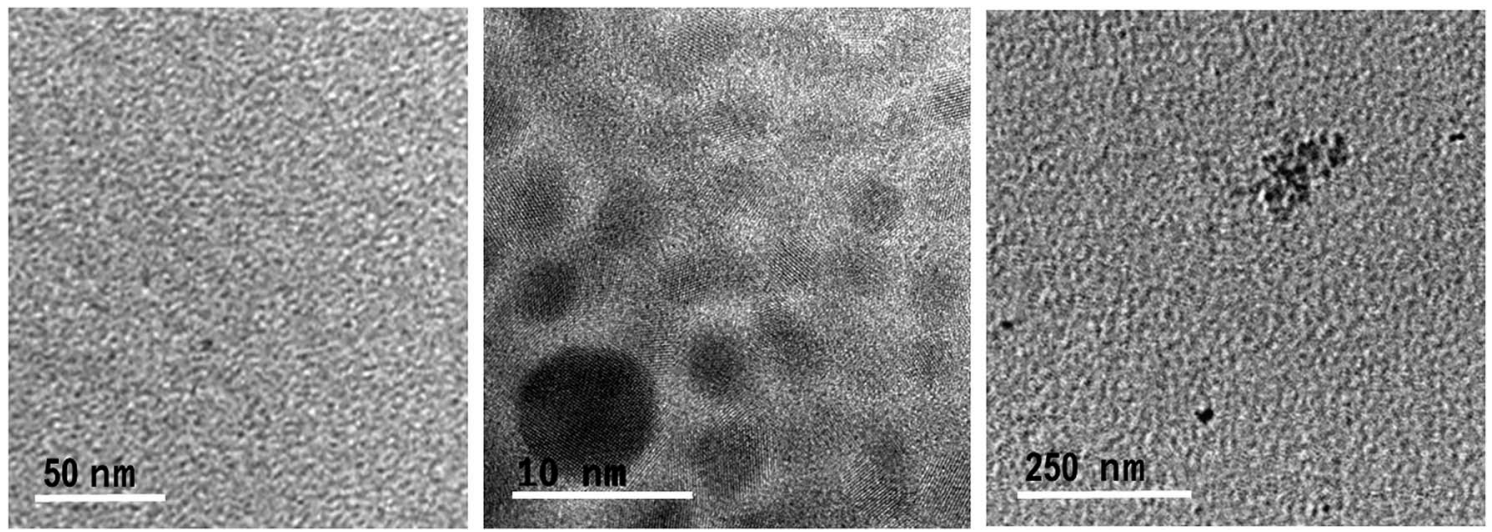

Fig. 3 TEM micrographs of individually and agglomerated GQDs derived from the GDQ-assembly (sample 5 of Table S1 $\uparrow$ ). 

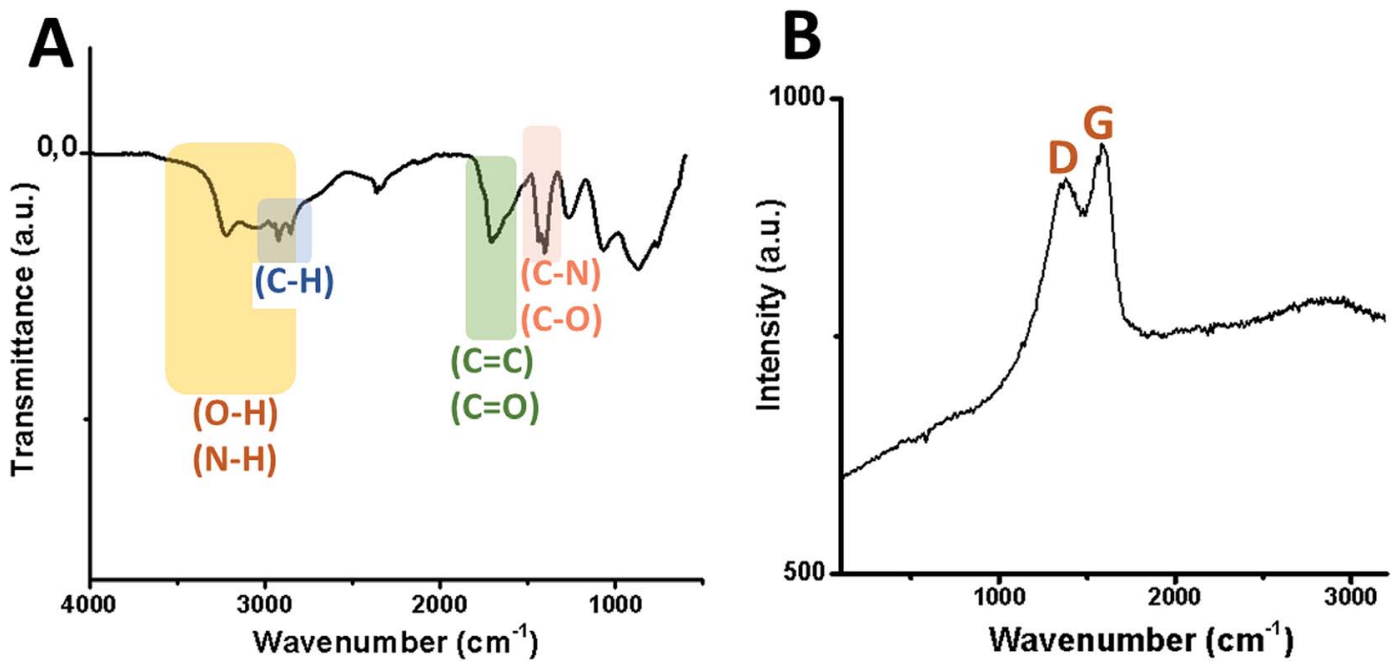

Fig. 4 Vibration modes of the infrared spectrum (A) and Raman shift (B) of GQDs derived from the GDQ-assembly (sample 5 of Table S1†).

however, this is not as high as in aerogels possibly due to the drying strategy which negative impact on the surface area. ${ }^{31}$ SEM analysis was performed to understand the external porous structure of the aerogels. As shown in Fig. 5, macropores within each group of flakes were observed.

Furthermore, after four months of storage under air conditions, the aerogel loses its robustness by starting getting wet and fluffy, being an indication of its large hygroscopic character. This destabilization can be explained by a strong water adsorption of the media after 60 days. Thus, these results indicate that the stability of GQD aerogel is mainly governed by hydrogen bonds rather than by hydrophobic interactions between $\pi$ layers of the nanodot, thus, being the edges containing oxygen and nitrogen groups formed during synthesis essential for building the porous GQD-architecture.

From the data, we proposed a mechanism for yielding the GQD-assemblies: the presence of $\mathrm{H}_{3} \mathrm{PO}_{4}$ catalyzes the dehydration process of the precursors along the microwave reaction promoting the carbonization and nucleation to achieve GQDs. By lowering the proportion of urea, the nitrogen content is also reduced at surface, and thus, the richer oxygen content due to carboxylic acids may be the promoter of the self-assembly into a gel-like structure at acidic $\mathrm{pH}$.
As a consequence of the potential risk of herbicides in the environment, methods for eliminating and monitoring such kind of contaminants are required. In this sense, the large surface area and high intensity photoluminescence of GQDs offer interesting possibilities for their application as multifunctional material in environmental remediation. Thus, we demonstrated the applicability of this material with double functions in both polar and apolar media.

In first place, the sorbent capacity of the aerogel was evaluated in apolar media using the family of triazines as representative herbicides (simetryn, prometryn, and terbutryn). GC/MS was used to demonstrate extraction capacity of the material towards the selected hazardous substances. The effect of the contact time (0-120 min) was first evaluated by direct immersion of the sorbent in solutions containing the selected analytes at a concentration of $1 \mathrm{mg} \mathrm{mL}{ }^{-1}$. Fig. 6 indicates that the complete extraction occurred after 60 min of contact. Noticeable, excellent batch-to-batch reproducibility of the extraction using the GQDs-assembly given from different synthetic batches were obtained, finding a relative standard deviation (RSD) of $6.3 \%$ in terms of recovery.

The adsorption isotherm (Fig. $55 \dagger$ ) was then obtained for terbutryn (at concentrations of $20-180 \mathrm{mg} \mathrm{L}^{-1}$ ) as the model

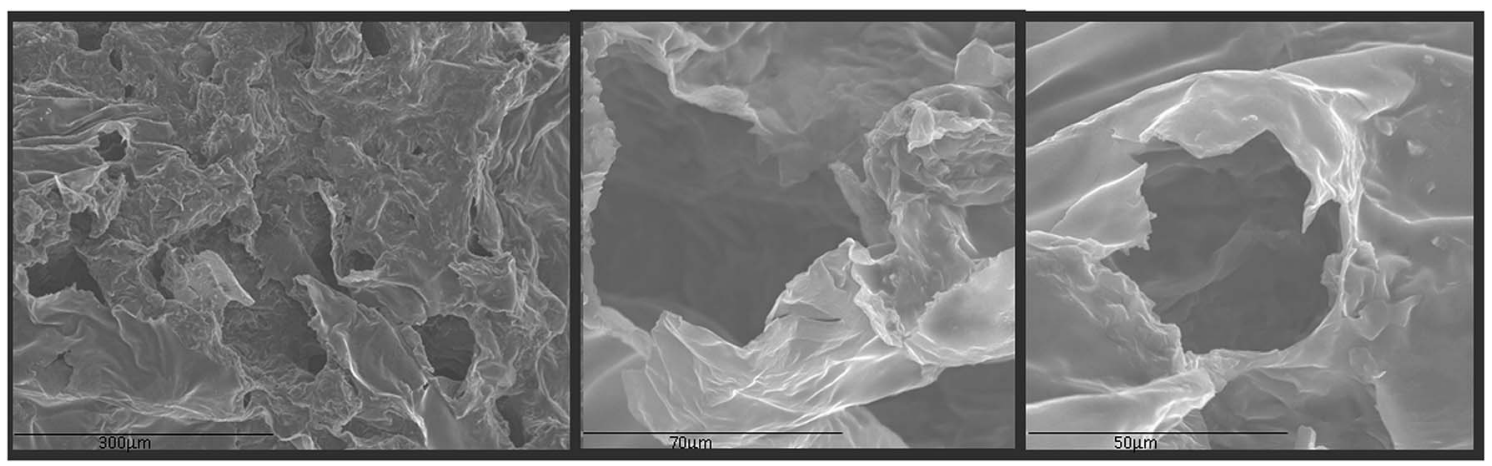

Fig. 5 SEM images of the GQD-assembly. 


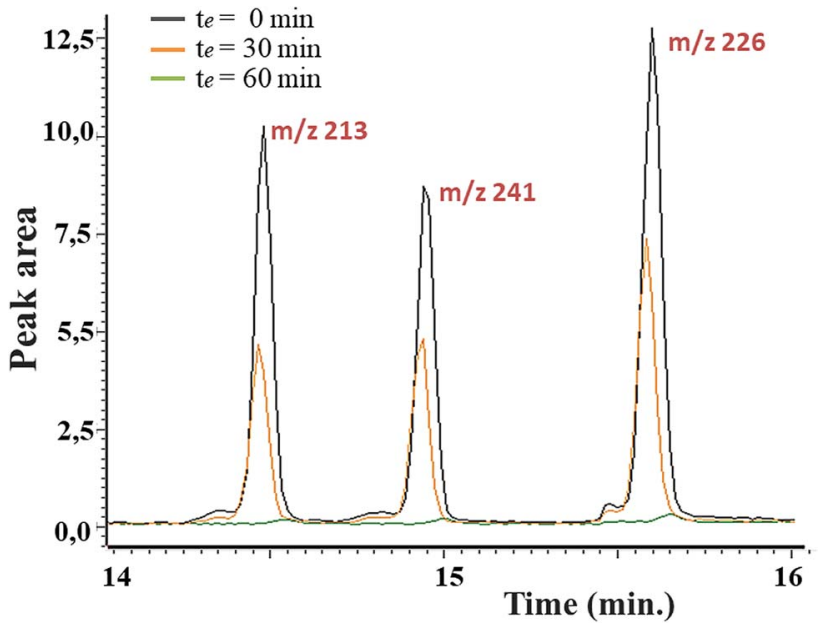

Fig. 6 Stacked chromatograms of different herbicides $(\mathrm{m} / \mathrm{z} 213$ for simetryn, $\mathrm{m} / \mathrm{z} 241$ for prometryn and $\mathrm{m} / \mathrm{z} 226$ for terbutryn) upon concentration of $1 \mathrm{mg} \mathrm{L}^{-1}$ and at different contact.

compound. The maximum loading capacity of nanostructured GQD-assembly resulted to be of $112 \mathrm{mg} \mathrm{g}^{-1}$ of sorbent, which is higher than other porous materials based on graphene $(27 \mathrm{mg}$ $\left.\mathrm{g}^{-1}\right)^{32}$ or GO aerogels (96-125 $\left.\mathrm{mg} \mathrm{g}^{-1}\right)^{33}$ and reduced GO hydrogels $\left(8-29 \mathrm{mg} \mathrm{g}^{-1}\right) .^{34}$ Typical capacity values for porous graphene assembled architectures spanned from $8 \mathrm{mg} \mathrm{g}^{-1}$ in $\mathrm{GO}^{34}$ to hundreds as occurred for hybrid aerogels of graphene combined with carbon nanotubes (CNTs). ${ }^{35}$ Other carbon aerogels using extra linkers such as CNT with dendritic poly(amido amine) molecules for adsorbing a cationic thiazine dye displayed adsorption capacities of 84 and $103 \mathrm{mg} \mathrm{g}^{-1}$ depending on the presence and absence of magnetic nanoparticles. ${ }^{36}$

Finally, our attention is paid to the chemical sensing of triazines in polar protic media. Noticeably, the optical properties of GQDs when dispersed in water, methanol and ethanol became very promising for the effective determination of triazines. Thus, the herbicide, being an electron-deficient heterocyclic system, is expected to quench the photoluminescence of GQDs acting as electron donor, by a photoinduced electron transfer process. Experiments were performed in ethanol, and the intensity of the characteristic emission of GQDs was found to be altered by the presence of the target molecule as quencher; in this case, the terbutryn produced fluorescence quenching of the graphene nanodots (Fig. S6A $\dagger$ ). Similar behaviour were found for simetryn and prometryn (Fig. S6B and $\mathrm{C} \dagger$ ), becoming very useful for monitoring the remediation efficiency. The fluorescence quenching may be due to the interaction between the electron-deficient triazine ring of the analyte and the electron-rich GQDs, possibly via $\pi$-stacking interactions, opening a quenching channel due to the electron transfer between GQDs and the target molecule. The quenching response of the fluorescence by terbutryn was fitted in the linear range of $0.8-20.7 \mathrm{nmol} \mathrm{mL}^{-1}$ to the Stern-Volmer equation, which is $\left(I_{0} / I\right)=0.0771 c+1.0891$, with a correlation coefficient of 0.992 as an indicative of the high detection accuracy of the method. To demonstrate the sensing method toward terbutryn, the detection and quantification limits, which are calculated as three times and ten times of the standard deviation of the blank divided by the slope of the calibration equation, respectively, resulted to be of 4.53 and $13.75 \mathrm{nmol} \mathrm{mL}{ }^{-1}$. Since most of carbon-based nanodots displayed fluorescence quenching by a wide range of metal ions as occurred for the ensuing GQDs (Fig. S7 $\dagger$ ), in this study, the use of ethylenediaminetetraacetic acid $(5 \mathrm{mM})$ as chelating agent was crucial to remove these interferences enabling the monitorization of the quenching process by herbicides at lower concentrations.

It is expected from these results that non-covalent interactions are responsible for the highly selective sorption of the terbutryn into the aerogel. In the gel-like state these noncovalent forces are composed by the mentioned $\pi$-stacking interactions and the strong hydrogen binding between the hindered amine groups of the terbutryn and the carboxyl groups of the GQDs.

While most of reports demonstrated the applicability of GQDs and its derivatives as sensors or as sorbents or as catalysts (Table S2 $\dagger$ ), ${ }^{1,6}$ in this work we make a step forwards demonstrating the two roles of the ensuing material in remediation; thus, both functions of such material (as excellent sorbent material and fluorescent sensor) meet all requirements to improve remediation activities by targeting the selected environmental contaminants.

\section{Conclusions}

We demonstrated an easy and fast way of preparing GQDassemblies using microwave irradiation from low-cost organic precursors as an ecofriendly one-pot alternative of conventional heating synthetic methods for both GQDs generation and self-assembly in one single step. The highly directed hydrogen bonds and $\pi$-stacking interactions are mainly responsible for the solid network formation. Thus, the porous GQD-assembly is mechanically robust in apolar solvents like hexane and chloroform, and resulted to exhibit a large sorption capacity versus triazine compounds like terbutryn. Terbutryn removal efficiency was proved with GQDassemblies obtained from different synthetic batches, confirming both the high sorption capacity and the excellent batch-to-batch reproducibility of the material.

Due to its hygroscopicity for the high content of oxygencontaining groups, the GQD-assemblies collapse in water under sonication waves resulting in the release of the individual nanosheets, which exhibited high emission. After the disassembling, the characteristic optical properties of isolated GQDs based on quantum confinement is restored in solution, being as a suitable manner for sensing the presence of triazines, as a result of the fluorescent quenching observed upon increasing the herbicide concentration. After observing these promising functions for the ensuing material, our efforts are now driven toward portable technologies capable of fluorescent monitoring triazines while extracting in the real world. The highly sorption abilities of such porous networks and the supreme bright fluorescence of GQDs once dissembled in polar solvents hold promises in both environmental and food sectors. 


\section{Conflicts of interest}

There are no conflicts to declare.

\section{Acknowledgements}

This research was supported by the Spanish Ministry of Innovation and Science for funding CTQ2017-83175R project. B. Fresco-Cala expresses her gratitude for the predoctoral grant (ref FPU13/03896) from the Spanish Ministry of Education. Also, the authors would like to thank the Central Service for Research Support (SCAI) of the University of Cordoba for the microscopes. A. S., M. C. and F. M. thank the LAMP group (www.unipa.it/lamp) at University of Palermo for support and stimulating discussions.

\section{References}

1 A. Cayuela, M. L. Soriano, C. Carrillo-Carrión and M. Valcárcel, Chem. Commun., 2016, 52, 1311-1326.

2 A. K. Geim and K. S. Novoselov, Nat. Mater., 2007, 6, 183-191. 3 L. A. Ponomarenko, F. Schedin, M. I. Katsnelson, R. Yang, E. W. Hill, K. S. Novoselov and A. K. Geim, Science, 2008, 320, 356-358.

4 Y. Du and S. Guo, Nanoscale, 2016, 8, 2532-2543.

5 P. N. Joshi, S. Kundu, S. K. Sanghi and D. Sarkar, in Smart Drug Delivery System, InTech, 2016.

6 S. Benítez-Martínez and M. Valcárcel, TrAC, Trends Anal. Chem., 2015, 72, 93-113.

7 B. Zheng, Y. Li and J. Liu, Appl. Phys. A, 2002, 74, 345-348.

8 J. Zou, J. Liu, A. S. Karakoti, A. Kumar, D. Joung, Q. Li, S. I. Khondaker, S. Seal and L. Zhai, ACS Nano, 2010, 4, 7293-7302.

9 L. Hough, M. Islam, B. Hammouda, A. Yodh and P. Heiney, Nano Lett., 2006, 6, 313-317.

10 R. Du, Q. Zhao, N. Zhang and J. Zhang, Small, 2015, 11, 3263-3289.

11 S. M. Arnold, W. J. Hickey, R. F. Harris and R. E. Talaat, Environ. Toxicol. Chem., 1996, 15, 1255-1262.

12 Y. Ji, C. Dong, D. Kong, J. Lu and Q. Zhou, Chem. Eng. J., 2015, 263, 45-54.

13 M. Sick, E. Alesi and G. Bott-Breuning, in Integrated Soil and Sediment Research: A Basis for Proper Protection, Springer, 1993, pp. 663-667.

14 T. Satapanajaru, P. Anurakpongsatorn, P. Pengthamkeerati and H. Boparai, Water, Air, Soil Pollut., 2008, 192, 349-359.
15 C. Ruiz-Palomero, M. L. Soriano, S. Benítez-Martínez and M. Valcárcel, Sens. Actuators, B, 2017, 245, 946-953.

16 C. Ruiz-Palomero, S. Benítez-Martínez, M. L. Soriano and M. Valcárcel, Anal. Chim. Acta, 2017, 974, 93-99.

17 L. Lv, Y. Fan, Q. Chen, Y. Zhao, Y. Hu, Z. Zhang, N. Chen and L. Qu, Nanotechnology, 2014, 25, 235401.

18 O. Chen, Y. Hu, C. Hu, H. Cheng, Z. Zhang, H. Shao and L. Qu, Phys. Chem. Chem. Phys., 2014, 16, 19307-19313.

19 X. Hou, Y. Li and C. Zhao, Aust. J. Chem., 2016, 69, 357-360. 20 D. Qu, M. Zheng, P. Du, Y. Zhou, L. Zhang, D. Li, H. Tan, Z. Zhao, Z. Xie and Z. Sun, Nanoscale, 2013, 5, 12272-12277. 21 Y. Kang, B. Yuan, D. Zhang, T. Ma, X. Huang, Z. Chu and K. Lai, J. Lumin., 2016, 179, 501-510.

22 H. Jiang, Y. Zhu, Y. Su, Y. Yao, Y. Liu, X. Yang and C. Li, J. Mater. Chem. A, 2015, 3, 12642-12645.

23 Z. Jin, P. Owour, S. Lei and L. Ge, Curr. Opin. Colloid Interface Sci., 2015, 20, 439-453.

24 A. Sciortino, A. Cayuela, M. L. Soriano, F. M. Gelardi, M. Cannas, M. Valcárcel and F. Messina, Phys. Chem. Chem. Phys., 2017, 19, 22670-22677.

25 E. LeCroy, F. Messina, A. Sciortino, C. E. Bunker, P. Wang, K. A. Shiral Fernando and Y.-P. Sun, J. Phys. Chem. C, 2017, 121, 28180-28186.

26 X. Wang, L. Cao, S.-T. Yang, F. Lu, M. J. Meziani, L. Tian, K. W. Sun, M. A. Bloodgood and Y.-P. Sun, Angew. Chem., 2010, 122, 5438-5442.

27 L. Tang, R. Ji, X. Li, K. S. Teng and S. P. Lau, J. Mater. Chem. C, 2013, 1, 4908-4915.

28 I. S. Amiinu, J. Zhang, Z. Kou, X. Liu, O. K. Asare, H. Zhou, K. Cheng, H. Zhang, L. Mai, M. Pan and S. Mu, ACS Appl. Mater. Interfaces, 2016, 8, 29408-29418.

29 R. Li, Z. Wei and X. Gou, ACS Catal., 2015, 5, 4133-4142.

30 Y. Liu, M. Xiang and L. Hong, RSC Adv., 2017, 7, 6467-6473.

31 Z.-S. Wu, A. Winter, L. Chen, Y. Sun, A. Turchanin, X. Feng and K. Müllen, Adv. Mater., 2012, 24, 5130-5135.

32 Q. Han, Q. Liang, X. Zhang, L. Yang and M. Ding, J. Chromatogr. A, 2016, 1447, 39-46.

33 W. Lv, C. Zhang, Z. Li and Q.-H. Yang, J. Phys. Chem. Lett., 2015, 6, 658-668.

34 J. N. Tiwari, K. Mahesh, N. H. Le, K. C. Kemp, R. Timilsina, R. N. Tiwari and K. S. Kim, Carbon, 2013, 56, 173-182.

35 Z. Sui, Q. Meng, X. Zhang, R. Ma and B. Cao, J. Mater. Chem., 2012, 22, 8767-8771.

36 X. Zhang, L. Chen, T. Yuan, H. Huang, Z. Sui, R. Du, X. Li, Y. Lu and Q. Li, Mater. Horiz., 2014, 1, 232-236. 